\title{
Artículo Transcultural
}

\section{El Análisis de Datos en la Investigación con El Método Fenomenológico}

Por:

Dra. Lydia Gordón de Isaacs. Departamento de Salud de Adultos. Facultad de Enfermería. Universidad de Panamá. Email: isaacs@cwpanama.net

\section{Resumen}

Este artículo se centra en el proceso de análisis de datos fenomenológicos, con una propuesta de modificación al modelo de análisis de Clark Moustakas (1994), presentando una discusión que busca fundamentar la propuesta. Se hace referencia a la vida del creador de la filosofía y método de investigación fenomenológica, Edmundo Husserl, y se introduce al filósofo Martín Heidegger quien amplío la investigación fenomenológica, fundamentando la interpretación de los datos fenomenológicos.

Palabras claves: Fenomenología, Análisis de Datos, Investigación.

\section{The Analysis of Data in Research with The Phenomenological Method}

\begin{abstract}
This article emphasizes the process of analysis of phenomenological data, with a proposal to modify Clark Moustakas (1994) analysis model, presenting a discussion to support the proposal. We make reference to the life of Edmund Husserl, creator of phenomenology as a philosophy and research Method and we introduce the philosopher Martin Heidegger, who extended phenomenological research with notion of interpretation as a fundamental aspect of phenomenological data analysis.
\end{abstract}

Key words: Phenomenology, Data Analysis, Research.

\section{A Análise de Dados em la Pesquisa com O Método Fenomenológico}

\section{Resumo:}

Este artigo enfoca o processo de análise de dados fenomenológicos, com proposta de modificação do modelo de análise de Clark Moustakas (1994), apresentando uma discussão que procura fundamentar a proposta.

Refere-se à vida do criador da filosofia e do método de pesquisa fenomenológica, Edmundo Husserl, e apresenta o filósofo Martin Heidegger que expandiu a pesquisa fenomenológica, com base na interpretação de dados fenomenológicos. 
Palavras Chaves: Fenomenologia, Análise de Dados, Pesquisa. Introducción

La fenomenología tuvo sus inicios a finales del siglo XIX, como parte del cambio paradigmático, que introducía a la investigación cualitativa como otra ruta para llegar al conocimiento científico.

Al filósofo Edmundo Husserl (1859- 1938) se le reconoce como el creador de la fenomenología, aunque la literatura indica que fue inspirado por las enseñanzas de Franz Brentano, filósofo distinguido de la época, quien fue su maestro.

Este artículo se centrará en el proceso de análisis de datos fenomenológicos, con una propuesta de modificación al modelo de análisis de Carl Moustakas (1994), presentando una discusión que busca fundamentar la modificación.

Dedicaremos un espacio breve para hacer referencia a detalles de la vida del creador de la filosofía y método de investigación que conocemos como fenomenología y debido a que el proceso de análisis tiene una etapa descriptiva y otra interpretativa, también introduciremos al filósofo Martín Heidegger quien amplío la investigación fenomenológica, introduciendo la interpretación de los datos fenomenológicos. Es decir, explica y fundamenta la necesidad de ir más allá de la descripción de los datos.

Antecedentes de Edmundo Husserl, nace en 1859 en Moravia, República Checa. Estudió en la Universidad de Viena donde obtuvo su PhD en Matemáticas, en 1883. Estando en Viena, por curiosidad fue a unas conferencias de psicología y filosofía que dictaba Franz Brentano, filósofo distinguido de la época y se sintió tan motivado, con las conferencias de Brentano, que decidió dedicarse al estudio de la Filosofía.

En 1887, Husserl fue contratado como profesor en la Universidad de Halle, y allí trabajó hasta 1916. Se cambia a la Universidad de Freiberg, donde trabajó hasta 1928 cuando se jubiló.

Fue autor de numerosos escritos, originales y profundos. Muchas de sus obras fueron destruidas durante la invasión de los nazis y Husserl fue perseguido, murió en 1938.

El padre Herman Leo van Breda, arriesgó su vida en 1939, llevando a Bélgica más de 40 mil hojas manuscritas, escondidas, por Husserl antes de morir.

La Fenomenología Trascendental de Husserl define la fenomenología como la filosofía descriptiva de las esencias de las experiencias puras.

Pretendía capturar las esencias de las experiencias, sin interpretar ni explicarlas, solo describiéndolas.

Señaló que había otras formas, no convencionales, o contrarias al paradigma prevalente, de llegar al conocimiento científico. La fenomenología era el método que permitiría llegar a ese conocimiento. 
Señaló que "El mundo está lleno de experiencias que nos pueden llevar a conocer científicamente los fenómenos".

En otras palabras, sostenía que "de la experiencia humana...vivida con los fenómenos...... podíamos extraer conocimientos científicos".

Era posible llegar a la esencia del fenómeno, a través del estudio sistemático de la experiencia vivida con el mismo.

La filosofía de Husserl evidentemente era existencialista y se define como descriptiva, eidética o trascendental. Sostenía que, mediante el estudio sistemático de las experiencias vividas con los fenómenos, podemos llegar a conocer el significado esencial de dicho fenómeno.

Se basó en las siguientes premisas: “...Cada experiencia puede ser sometida a la reflexión, y precisamente, mediante la reflexión...nos hacemos conscientes de la experiencia...".

"...Cotidianamente vivimos experiencias, pero solo cuando nos detenemos a reflexionar sobre ellas, nos percatamos de lo vivido..." "...A través de la intencionalidad podemos dirigir nuestra conciencia a determinados fenómenos..." "...Podemos llegar a la esencia de los fenómenos a partir del análisis de la experiencia vivida con el fenómeno..." "....Solo los que han vivido una experiencia la pueden describir..." "...No se puede aceptar un juicio como científico si no ha sido derivado de la evidencia de las experiencias...”.

La fenomenología ontológica o interpretativa de Martín Heidegger nació en una pequeña Villa en Alemania, el 26 de septiembre de 1889, fue a una escuela de gramática donde su maestro, le habló de Brentano y le regaló un libro de ese autor.

Heidegger empezó a estudiar teología en 1909 hasta 1911...pero después decidió cambiarse al campo de matemáticas y filosofía.

Heidegger fue discípulo de Husserl, pero pensó la fenomenología de manera diferente a su maestro. Para él no era solo describir el fenómeno, era necesario comprenderlo e interpretarlo. Sostenía que experiencias humanas se tienen que interpretar no solo describir, para llegar al verdadero significado.

Para Heidegger la vuelta al mundo de las experiencias se volvió más que un proyecto epistemológico para saber cómo es el fenómeno. En lugar de preguntar cómo es la esencia del fenómeno sostenía que debíamos preguntar cómo el "Ser" de las cosas se nos muestra.

El foco de Heidegger era encontrar el significado del Ser o del fenómeno. Pero además tenía otras preocupaciones, pensaba en nuestra temporalidad en el mundo.... Nuestra dinámica de cambios continuos durante nuestra estadía en el mundo.

Cuando describimos algo asumimos que es permanente, pero nada es siempre igual o incambiable. La experiencia cambia con el tiempo. 
Por lo tanto, no se trata de describir solamente. Heidegger habla de conectarnos con el mundo, ser parte del mundo, la fenomenología es entrar en el mundo de las experiencias, cuestionarlo y buscar sus secretos.

La fenomenología como método de investigación para Husserl y también para Heidegger, la fenomenología es una ciencia rigurosa que estudia los fenómenos del mundo. En sus meditaciones cartesianas declara la importancia del principio metodológico:

“...Es claro que no puedo aceptar un juicio como científico si no es derivado de la evidencia de las experiencias...” (Husserl,1977). “...Los fenómenos transcendentales son las entidades de las experiencias en el mundo, que se convierten en objetos de nuestra reflexión para encontrar sus significados..." (Husserl, 1977). En otras palabras, mediante el método fenomenológico podemos estudiar las experiencias de las personas, con el fenómeno que deseamos estudiar y de esta manera llegar al significado esencial del fenómeno (Husserl), o llegar a comprender y a interpretar el significado del fenómeno (Heidegger).

De acuerdo a Van Manen (2014), la fenomenología es parte de las ciencias humanas y se diferencia de las ciencias naturales, en que esta última, estudia objetos de la naturaleza y la forma como cambian las cosas, mientras que la ciencia humana estudia a las personas o seres que tienen conciencia y que actúan con un propósito dentro y sobre el mundo. El método de las ciencias humanas dice, envuelven descripción, análisis reflexivo, análisis crítico, interpretación.

Dilthey (1976), en ese sentido dice “...explicamos la naturaleza, pero la vida humana, la debemos entender...".

Es claro entonces que, como investigadores, la técnica de la entrevista en profundidad a los participantes que han vivido la experiencia, nos llevará a la colección de los datos con el método de investigación fenomenológica.

Por otro lado, mediante el análisis de los datos transcritos de la entrevista, llegaremos al carácter trascendental o escondido del fenómeno estudiado.

Para ello, el investigador debe suspender sus prejuicios, conocimientos previos y teorías, para enfocarse en los datos obtenidos de la entrevista. Husserl trató de demostrar que con el "époche" las cosas que se tienen en la conciencia se pueden suspender o poner entre paréntesis. Para enfocarnos en la experiencia que se describe. Mediante la reducción se puede llegar a la esencia fundamental, a la estructura vital, de los fenómenos del mundo. En cuanto a la intencionalidad sostenía Husserl sostenía que, el investigador, Intencionalmente puede guiar su mente, para que ponga entre paréntesis todos los conocimientos, teorías, prejuicios sobre un fenómeno y dirigir la consciencia a la experiencia vivida con un fenómeno.

También señaló que a través de la reducción de los datos se puede llegar a la esencia del fenómeno....al significado de los fenómenos del mundo. 
Es decir, el análisis de los datos nos permite llegar al significado esencial del fenómeno, a su estructura o componentes esenciales, por ello, la frase "volver a las cosas mismas" se ha convertido en la frase de la fenomenología transcendental.

El análisis de datos en la investigación fenomenológica

Recordemos que la entrevista fenomenológica debe buscar la descripción de las experiencias de los participantes, quienes fueron seleccionados por haber vivido la experiencia con el fenómeno de estudio. Por lo tanto, las preguntas del investigador son importantes, ya que determinan la calidad de los datos que se obtendrán, desde la pregunta central hasta la última pregunta colateral o secundaria.

Luego de concluir la entrevista, esta se transcribe. Existen diferentes abordajes o modelos para el análisis de los datos fenomenológicos, hemos utilizado el modelo de Clark Moustakas (1994), el cual es el resultado de dos modificaciones previas, ya que este autor abrevió el método de Van Kaam $(1959 ; 1966)$, y después el método de Stevick - Colaizzi Keen, (1973) y propuso los siguientes pasos para el análisis de los datos fenomenológicos:

1. Lectura total de la transcripción.

2. Segunda lectura de la transcripción, para extraer unidades de significado. Subrayando y después enlistando las unidades de significado (palabras o frases significativas relacionadas al fenómeno de estudio), para crear las categorías.

3. Análisis de las unidades de significado (buscando convergencias o similitudes entre las categorías).

4. Agrupación de unidades de significados para la formación de temas centrales.

Estos pasos se realizan con cada entrevista de acuerdo al modelo de Moustakas (1994), luego de obtener los temas centrales de cada entrevista se procede con el grupo de temas al paso siguiente.

5. Se realiza una descripción textural: Se realiza a partir de los temas centrales de cada entrevista. Esta descripción trata de presentar un panorama general del fenómeno a partir de los temas centrales.

6. La descripción estructural: Este paso se realiza para cada entrevista. Se determina la estructura de cada tema o atributos esenciales y estos pueden interpretarse en relación a los temas fenomenológicos universales.

7. La descripción textural-estructural: Este paso se realiza para cada entrevista. Se hace una descripción global integrando las dos descripciones anteriores, tanto la textural como la estructural.

8. Al final se integran todas las descripciones, texturales, estructurales y compuestas de todas las entrevistas.

a. Se unen todas las descripciones texturales en una sola descripción textural.

b. Se unen todas las descripciones estructurales en una sola descripción estructural

c. Se unen todas las descripciones compuestas en una sola descripción y tendremos una descripción integral textural-estructural.

La modificación del modelo de Moustakas. La modificación propuesta por (Gordón de Isaacs, 2017) consiste en un abordaje que inicia igual con los pasos 1,2,3, y 4, se van identificando los temas centrales de cada entrevista hasta lograr la saturación. En ese momento ya no se sigue entrevistando y 5. Con el grupo final de temas centrales, se realiza 
la descripción textural (un retrato de la experiencia que incluye sentimientos, y sensaciones. 6. la descripción estructural (las estructuras o características existenciales universales de la experiencia) y 7. la descripción compuesta (combinando la descripción textural con la estructural.

Sostenemos que los temas centrales globales son el producto integral del estudio. Allí se integran los temas de cada entrevista, en un solo producto, por lo tanto, las descripciones textural, estructural y compuesta final serán también productos integrales que incluirán todos los temas que identificamos por saturación de todas las entrevistas.

La saturación es la técnica que nos permite saber cuándo tenemos los datos "completos", del fenómeno estudiado y por lo tanto dejamos de incluir otros participantes en el estudio. Con estos datos "completos" realizamos las descripciones e interpretaciones.

Este concepto de saturación es lo que fundamenta nuestra propuesta ya que lo que corresponde es trabajar con los temas que han surgido de todas las entrevistas, para realizar la descripción textural, estructural y compuesta del estudio. Cuando saturamos sabemos que tenemos todos los temas y, por tanto, las unidades de significado del fenómeno de estudio. Estos temas son los completos, los globales, el resultado de cada entrevista, se considera como aproximaciones o sea que no nos dan el panorama integral.

En otras palabras, se hace una sola descripción textural, estructural, y compuesta a partir de los temas centrales identificados como producto de todas las entrevistas.

En nuestra experiencia con el modelo modificado, los temas que emergen de cada entrevista, van pareciéndose más y más, hasta coincidir con los temas centrales finales.

La reducción eidética de Husserl es el centro de la reflexión en su fenomenología transcendental, busca describir aquello que se muestra a nuestra conciencia de la experiencia y cómo se nos muestra. La reducción eidética se enfoca en aquello que es distinto o único de la experiencia. Nos permite identificar los significados y describir los temas centrales de la experiencia.

En términos existenciales podemos decir que la experiencia vivida, se da en un tiempo y en un espacio dado, por lo que Heidegger (1977), señala que es necesario preguntar ¿dónde y cómo está lo vivido en tiempo y espacio?. ¿Cómo está corporalmente, y en relación al mundo, a los otros y quien vive la experiencia? ¿Cómo es la relación con las cosas materiales del mundo?

La fenomenología se enfoca en las experiencias vividas, pero la reflexión fenomenológica empieza cuando como investigadores tratamos de captar los significados de la experiencia.

La fenomenología como ciencia, es el estudio de las experiencias vividas o de los significados existenciales, intenta describir e interpretar estos significados en detalle y profundidad.

Esta reducción de la experiencia requiere que se evite toda abstracción, teorización y generalización previa. 
Por supuesto, que todo proyecto de investigación debe examinar las teorías existentes y discutir el cuerpo de conocimiento existente acerca del fenómeno. Esta discusión se puede incorporar al finalizar la descripción e interpretación fenomenológica.

La metodología fenomenológica tiene desafíos porque se argumenta que podemos incorporar nuevas técnicas y estrategias metodológicas, pero sostiene a la vez que no podemos reducir la investigación a un grupo de estrategias generales (Heidegger, 1977).

Por otro lado, se enfatiza que se debe experimentar metodológicamente, pero de manera bien informada. La reducción fenomenológica de los datos no es un fin en sí, es más bien un medio para llegar a un fin: el fin es poder entender el mundo tal como se vivió. Los métodos nos dan un mapa parcial para llegar al destino. La experiencia, es una forma profunda y enriquecida de entender el mundo vivido (van Manen, 2014).

El análisis fenomenológico debe ser realizado con experiencias pre-reflexivas. No puede realizarse en puntos de vista, opiniones, creencias, percepciones o interpretaciones de la experiencia de los participantes. No debe confundirse las descripciones concretas de las experiencias vividas con las interpretaciones de las experiencias.

Los temas fenomenológicos universales. Una forma de asistir en el proceso de reflexión fenomenológica es usando los temas fenomenológicos universales relacionados a las experiencias vividas. Estos son: relaciones vividas (relaciones con uno mismo o con otros), corporalidad vivida (el cuerpo), espacio vivido (el espacio o contexto), tiempo vivido (temporalidad) y cosas materiales o tecnológicas vividas (materialismo).

Estos tópicos fenomenológicos pertenecen o están presentes en toda experiencia humana, son temas universales de vida (van Manen, 2016). Estos existenciales fundamentales ocurren repetidamente en la literatura fenomenológica. Todos experimentamos nuestro mundo y nuestra realidad mediante estos existenciales, Yo soy parte del mundo, mi cuerpo es una forma de comunicarme con el mundo (Merleau Ponty, 1962).

Yo puedo describir mi relación con el otro, puedo percibir al otro (Sartre, 1965). La existencia es antes que la esencia (Sartre,1965). Las personas estamos inmersas en el mundo, no somos criaturas aisladas. Estamos en el mundo en relación a otros (Heidegger, 1977). Por lo tanto, el análisis de los datos fenomenológicos incluye la descripción e interpretación de las experiencias y sus significados.

\footnotetext{
Referencias

Colaizzi, P. (1973). Reflections and Research in Psychology; a Phenomenological Study of Learning. Dubuque, IOWA: Kendal Hunt. Pub. Co. Recuperado de: http://www.worldcat.org/title/reflection-andresearch-in-psychology-a-phenomenological-study-of-learning/oclc/762820
}

Rickman, H. P. (1976). Dilthey: Selected Writtings: Cambridge University Press. Recuperado de: https://www.amazon.in/Dilthey-Selected-Writings-H-Rickman/dp/0521209668

Giorgi, A. (1985). Phenomenology and psychological research. Pittsburgh: Duquesne University Press. Recuperado de: https://es.scribd.com/doc/45935704/Giorgi-s-Empirical-Phenomenological-Model 
Enfoque, Revista Científica de Enfermería, VOL. XXI, N 17 Julio a Diciembre 2017 ISSN 1816-2398 Impreso

Heidegger, M. (1977). Basic Writtings. New York: Harper and Row. Recuperado de: https://kinasevych.ca/2014/10/03/heidegger-1977-the-question-concerning-technology/

Husserl, E. (1970). Logical Investigations. (J.N. Findlay. Trans). New York: Humanities Press. Recuperado de:

https://www.ucd.ie/t4cms/Editors\%20IntroductionHusserlLogical-Investigations\%202001.pdf

Husserl, E. (1977). Cartesian Meditations: An Introduction to Metaphysics (D. Cairns, Trans.). The Haugue: Martinus Nijhoff. Recuperado de: http://www.scielo.cl/scielo.php?script=sci_arttext\&pid=S004934492006000300008

Keen, E. (1975). Doing Research Phenomenologycally. Unpublished Manuscript, Bucknell University, Lewisburg, PA. Recuperado de: https://acrwebsite.org/search/view-conference-proceedings.aspx?Id=6450

Merleau Ponty, M. (1962). Phenomenology of Perception (C. Smith, Trans.). Boston: Routledge and Kegan Paul. Recuperado de: https://link.springer.com/chapter/10.1007/978-90-481-2831-0_22

Moustakas, C. (1994). Phenomenological Research Methods. Thousand Oaks, California: Sage Publications, Inc. Recuperado de: https://www.amazon.es/Phenomenological-Research-Methods-ClarkMoustakas/dp/0803957998

Sartre, J. (1965). Introduction: The Pursuit of Being. In Being and Nothingness New York: Citadel Press. Recuperado de: https://plato.stanford.edu/entries/sartre/

Stevick, E. (1971). An Empirical Investigation of the Experience of Anger. In A. Giorgi, W. Fisher, and R. Von Eckartsberg (Eds), Duquesne Studies in Phenomenological Psychology (Vol. 1, pp. 132-148). Pittsburgh: Duquesne University Press. Recuperado de:

https://books.google.com.pa/books?id=2KXHhNg7ZfUC\&pg=PA58\&lpg=PA58\&dq=Stevick,+E.++(1971)

Van Kaam, A. (1966). Application of the phenomenological method. In A. Van Kaam, Existential foundations of psychology. Garden City, New York: Double-day. Recuperado de:

https://books.google.com.pa/books?id=pqgACAAAQBAJ\&pg=PA24\&lpg=PA24\&dq=Van+Kaam,+A.+(196 $6 \&$ source $=$ bl\&ots $=$ M4DIZ3Jr1u\&sig=wS3ZkO4GQMsu7

Van Manen, M. (2014). Phenomenology of Practice: Meaning giving methods in Phenomenology. Walnut Creek, CA: Left Coast Press, Inc. https://books.google.com.pa/books?id=50h-

CwAAQBAJ\&pg=PA98\&dq=Van+Manen,+M.++2014\&hl=es\&sa=X\&ved=0ahUKEwiDnrm0sqrXAhXK4i YKHf_IACQQ6

Van Manen, M. (2016). Researching Lived Experience. 2nd Ed. New York: Routledge Taylor \& Francis Group.

https://books.google.com.pa/books?id=IbhmDAAAQBAJ\&printsec $=$ frontcover\&dq=Van+Manen, + M. ++201

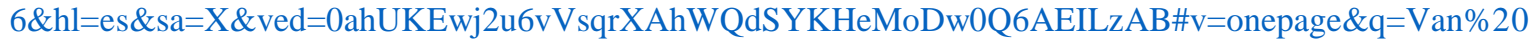
Manen\%2C\%20M.\%20\%202016\&f=false

Recibido: 12 de Agosto 2017

Aprobado: 30 de Noviembre 2017 